\title{
Sensitization of human colon adenocarcinoma cells (LoVo) to reactive oxygen species by a lysosomotropic compound
}

\author{
ENZO AGOSTINELLI $^{1}$, LAURA DALLA VEDOVA ${ }^{1}$, FRANCESCA BELLI $^{1}$, \\ MARIA CONDELLO ${ }^{1}$, GIUSEPPE ARANCIA ${ }^{2}$ and NIKOLAUS SEILER ${ }^{3}$ \\ ${ }^{1}$ Department of Biochemical Sciences 'A. Rossi Fanelli', University of Rome 'La Sapienza' and CNR, \\ Biology and Molecular Pathology Institutes, Rome; ${ }^{2}$ Department of Technology and Health, \\ Istituto Superiore di Sanità, Rome, Italy; ${ }^{3}$ Laboratory of Nutritional Cancer Prevention, ULP-EA3430, \\ Institut de Recherche contre les Cancers de l'Appareil Digestif (IRCAD), Strasbourg, France
}

Received December 28, 2005; Accepted March 27, 2006

\begin{abstract}
The in situ formation of cytotoxic metabolites by an enzyme-catalyzed reaction is a recent approach in cancer therapy. The present results show that multidrug-resistant human colon adenocarcinoma cells (LoVo) are significantly more sensitive than corresponding wild-type cells to hydrogen peroxide and aldehydes, the products of bovine serum amine oxidase (BSAO)-catalyzed oxidation of spermine. Pretreatment of the cells with $\mathrm{N}^{1}, \mathrm{~N}^{4}$-bis(2,3-butadienyl)-1,4butanediamine (MDL 72527), a lysosomotropic compound, sensitized both cell lines to the subsequent exposure to spermine metabolites, as was evident from the decrease of cell survival by a log unit. The sensitizing effect was greater in the case of the multidrug-resistant cell line, an aspect of particular importance with respect to potential therapeutic applications of the method, since conventional cancer therapy suffers from the development of drug resistance. Cell viability was determined using a clonogenic assay. MDL 72527 (at $300 \mu \mathrm{M}$ ) produced numerous cytoplasmic vacuoles, presumably of lysosomal origin, after 6-h exposure, which decreased in size and number (in the presence of the drug) by $24 \mathrm{~h}$ and had almost disappeared completely at $48 \mathrm{~h}$. Mitochondrial damage, as observed by transmission electron microscopy, seemed to correlate better with the cytotoxic effects of the treatment than the formation of vacuoles. We suggest that the release of lysosomal enzymes into the cytosol by MDL 72527 is the main reason for its sensitizing effect. It is known that lysosomotropic compounds, which release lysosomal enzymes, produce oxidative stress and apoptosis.
\end{abstract}

Correspondence to: Dr Enzo Agostinelli, Department of Biochemical Sciences 'A. Rossi Fanelli', University of Rome 'La Sapienza', Piazzale A. Moro 5, I-00185 Rome, Italy

E-mail: enzo.agostinelli@uniroma1.it

Key words: bovine serum amine oxidase, colon adenocarcinoma cells, polyamines, MDL 72527, multidrug resistance

\section{Introduction}

The so-called polyamines (putrescine, spermidine, spermine, and related structures) are simple aliphatic amines with numerous functions in cell biology (1). The deregulation of their metabolism may induce apoptosis (2). Apart from being of vital importance for the propagation and viability of most cells, the natural polyamines, spermidine and spermine, are also the source of cytotoxic metabolites. Their oxidative deamination generates hydrogen peroxide, aldehydes and ammonia. Fig. 1 shows the reaction of spermine with bovine serum amine oxidase (BSAO: EC 1.4.3.6). Hydrogen peroxide and the aldehydes induce apoptotic and non-apoptotic cell death; exposure of tumor cells to purified BSAO and spermine caused a time-dependent decrease of cell viability (3) and impaired the growth of a mouse melanoma (4). Formation of hydrogen peroxide by the xanthine oxidase reaction within a muscle also had an anti-tumor effect (5). Other experiments demonstrated that multidrug resistant (MDR) LoVo cells (obtained by exposure to doxorubicin) (6) are more sensitive to toxic spermine metabolites than their wild-type counterparts $(3,7)$.

Hydrogen peroxide is a major component of endogenous reactive oxygen species (ROS). It has multiple physiological functions in signal transduction cascades, and plays a role in the pathology of several disorders, including cancer and neurodegenerative diseases (8). Under the experimental conditions used in the present study, hydrogen peroxide is mainly responsible for cell damage; the spermine-derived aldehydes contribute to cytotoxicity by approximately $20 \%(3,9)$.

We are currently studying drug combinations with the aim to improve the induction of cell death by toxic polyamine metabolites. We describe results of combined treatments with BSAO/spermine with MDL 72527 ( $\mathrm{N}^{1}, \mathrm{~N}^{4}$-bis(2,3-butadienyl)1,4-butanediamine dihydrochloride). MDL 72527 is an inactivator of FAD-dependent polyamine oxidase. It has cytotoxic properties which are, however, unrelated to its ability to inactivate polyamine oxidase (10). This drug was chosen because it is lysosomotropic (11). We expected that, owing to its different toxic mechanism, it might amplify hydrogen-peroxide and aldehyde-induced cell death. 

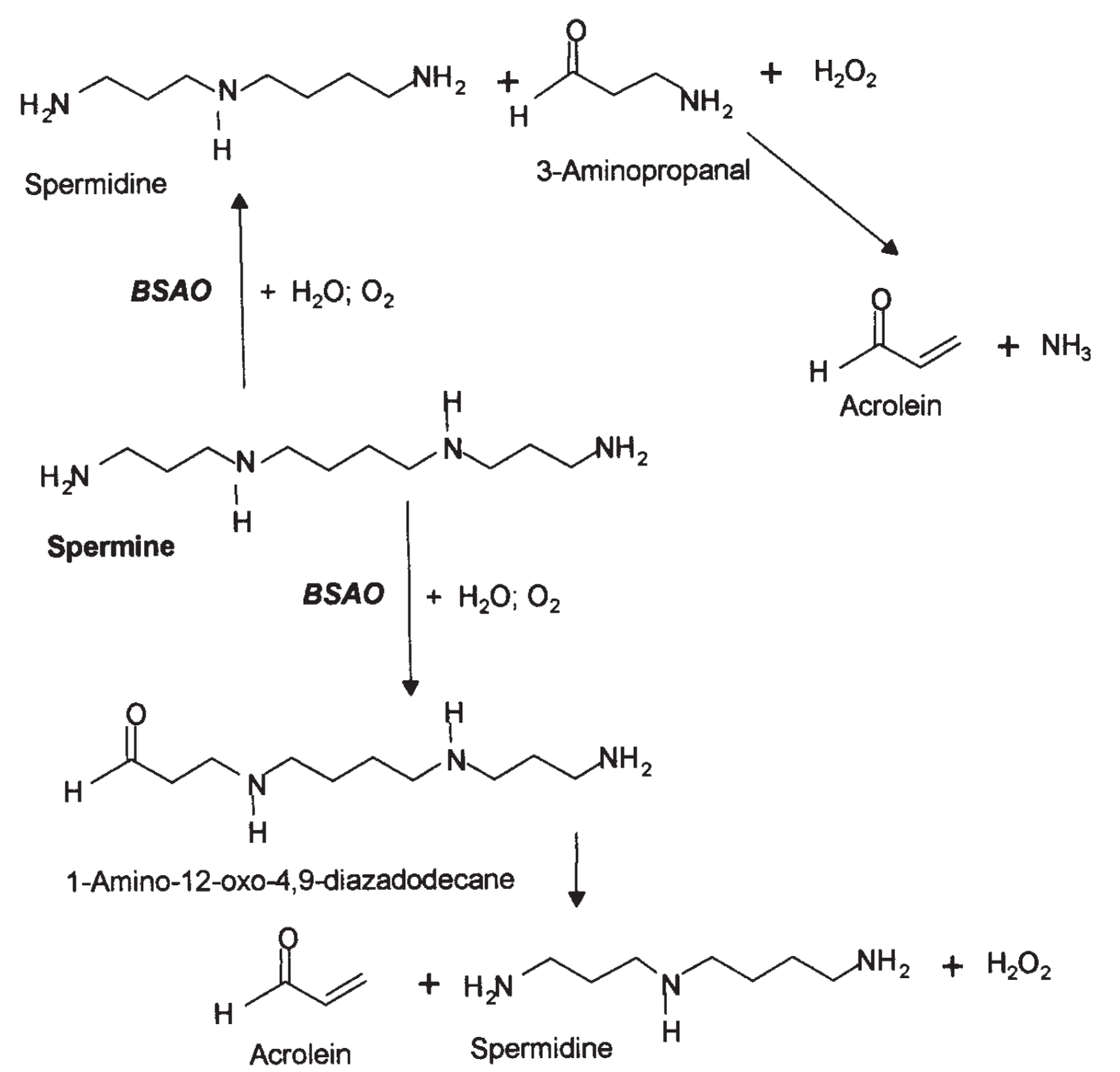

Figure 1. Metabolite formation from spermine by bovine serum amine oxidase (BSAO)-catalyzed reactions. The aldehydes that are preferentially formed under the reaction conditions (see Materials and methods) have not been identified. The monoaldehyde, 1-amino-12-oxo-4,9-diaza-dodecane, may be further oxidized to a dialdehyde (1,12-dioxo-4,9-diaza-dodecane) (not shown). Spermidine is a substrate of BSAO and will therefore contribute to hydrogen peroxide and aldehyde formation. Acrolein formation is a spontaneous process. The factors favoring its elimination from the oxidation products of spermine and spermidine have not been identified. The direct oxidation of spermine to spermidine and 3-aminopropanal by BSAO was reported by Houen et al (26).

\section{Materials and methods}

Materials. If not stated otherwise, usual laboratory chemicals were from Sigma Chemical Co., St. Louis (MO, USA). Spermine tetrahydrochloride was from FLUKA (Buchs, Switzerland), cell culture materials were from Gibco Life Technologies Ltd. (Paisley, UK). BSAO was purified to homogeneity essentially as described by Turini et al (12). The specific activity of the enzyme preparation was $0.38 \mathrm{IU} / \mathrm{mg}$ $\left(1 \mathrm{IU}=1 \mu\right.$ mole benzylamine oxidized per $\min$ at $25^{\circ} \mathrm{C}$ ). MDL 72527 was synthesized as described earlier (13).

Cell cultures. The human colon adenocarcinoma cell line (LoVo WT) (originally isolated from a metastatic nodule), was a gift from Professor E. Dolfini, Istituto per la Cura dei Tumori, Milan, Italy. The pleiotropic multidrug-resistant (MDR) cell line, LoVo DX, was selected for resistance to doxorubicin from its drug-sensitive parental cell line (6). LoVo DX cells are also resistant to other chemotherapeutic agents such as etoposide and vincristin (14). Cells were grown in monolayer in Ham's F12 medium with $10 \%$ fetal bovine serum (Hyclone, Europe Ltd., Cramlington, UK), $1 \%$ L-glutamine, penicillin $(50 \mathrm{U} / \mathrm{ml})$-streptomycin $(50 \mu \mathrm{g} / \mathrm{ml})$ and $1 \%$ vitamins in a humidified atmosphere of $5 \% \mathrm{CO}_{2}$ in a water-jacketed incubator at $37^{\circ} \mathrm{C}$.
Treatments and cell survival experiments. Cell survival experiments were carried out using confluent cells that had been incubated for $24 \mathrm{~h}$ or $48 \mathrm{~h}$ at $37^{\circ} \mathrm{C}$ with fresh culture medium containing MDL 72527 at concentrations in the range of 200-400 $\mu \mathrm{M}$. Cells were washed with $10 \mathrm{mM}$ EDTA in phosphate-buffered saline (PBS) and were then trypsinized by addition of $0.25 \%$ trypsin solution in PBS, washed with PBS supplemented with $1 \%$ bovine serum albumin (PBS/BSA), and pelleted by centrifugation ( $2 \mathrm{~min}$, $1500 \mathrm{x}$ g). The cells were resuspended in PBS/BSA. Freshly harvested LoVo cells $\left(10^{5} / \mathrm{ml}\right)$ were incubated at $37^{\circ} \mathrm{C}$ for different time intervals in the presence or absence of BSAO $\left(6.5 \times 10^{-3} \mathrm{IU} / \mathrm{ml}\right)$ and spermine. The spermine solution was freshly prepared before each experiment. After incubation, the cells were centrifuged, washed twice in PBS-BSA, and resuspended in $1 \mathrm{ml}$ PBS-BSA.

Cytotoxicity was evaluated by a plating efficiency assay, which determines the ability of the cells to form macroscopic colonies ( $>50$ cells). Aliquots of suspensions of $10^{5}$ cells $/ \mathrm{ml}$ were plated in tissue culture dishes $(50 \mathrm{~mm}$ diameter $)$ containing $5 \mathrm{ml}$ complete culture medium, and incubated at $37^{\circ} \mathrm{C}$. After 21 days, the colonies were fixed with $96 \%$ ethanol, stained with methylene blue and counted (three or four plates of each treatment). Mean values were calculated from the results of three independent experiments. Control 


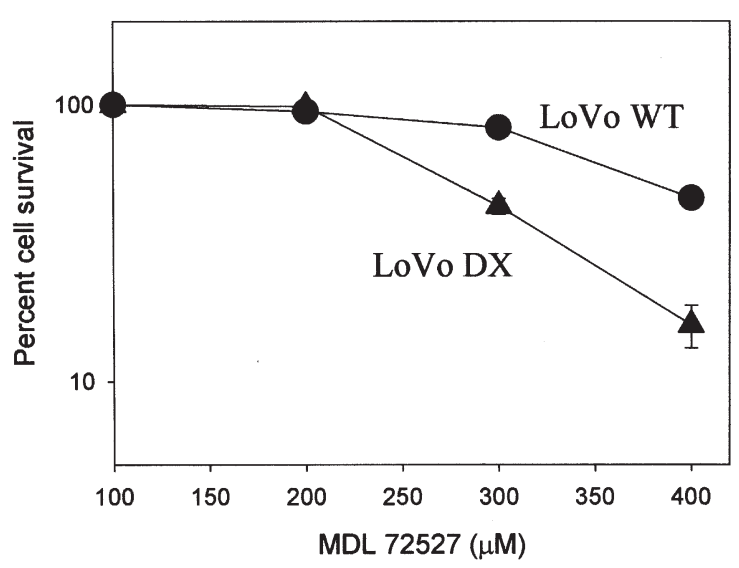

Figure 2. Effect of MDL 72527 on the survival of LoVo WT and LoVo DX cells. Dose-effect relationship for $24-\mathrm{h}$ incubation at $37^{\circ} \mathrm{C}$. Each point is the mean value of the results of three or four experiments with 2-5 samples per treatment. The error bars indicate \pm SD. When not shown, SD is smaller than the symbols.

plating efficiencies were higher than 85 and $80 \%$ for LoVo WT and LoVo DX cells, respectively. Percent cell survival was determined as the ratio between the mean number of colonies in treated and control samples.

Scanning electron microscopy. For scanning electron microscopy (SEM), cells were grown to near confluence on glass coverslips in Ham's F12 medium containing MDL 72527 at $200-400 \mu \mathrm{M}$ and $1 \% \mathrm{BSA}$, in the absence of serum, at $37^{\circ} \mathrm{C}$ for different times in the presence or absence of BSAO $\left(6.54 \times 10^{-3} \mathrm{IU} / \mathrm{ml}\right)$, and spermine $(6 \mu \mathrm{M})$. After incubation, cells were washed with F12 medium and were then processed for SEM as was described (15). The samples were examined by a Cambridge Stereoscan 360 scanning electron microscope (Cambridge Instruments, Cambridge, UK).

Transmission electron microscopy. For transmission electron microscopy (TEM), cells were grown to near confluence and harvested as described above, washed with PBS-BSA, centrifuged and resuspended in $2 \mathrm{ml}$ of F12 medium-1\% BSA, without serum. After incubation for $60 \mathrm{~min}$ at $37^{\circ} \mathrm{C}$ in the presence or absence of BSAO $\left(6.54 \times 10^{-3} \mathrm{IU} / \mathrm{ml}\right)$, or with spermine alone $(6 \mu \mathrm{M})$, cells were washed with F12 medium and then processed for TEM observation as previously described (15). Ultrathin sections were examined by a Zeiss EM 10C electron microscope (Zeiss, Oberkochen, Germany).

\section{Results}

Effect of pre-incubation with MDL 72527 on the plating efficiency of LoVo cells after exposure to BSAO and spermine. Both LoVo WT and LoVo DX cells were pre-incubated for $24 \mathrm{~h}$ with MDL 72527 at concentrations ranging from 100 to $400 \mu \mathrm{M}$ and, after washing, were exposed for up to $1 \mathrm{~h}$ to $\mathrm{BSAO}$ and spermine (at concentrations ranging from 0 to $15 \mu \mathrm{M}$ ) (for more details of the experimental procedure see Materials and methods).

It is evident from previous experiments (3), as well as from control experiments carried out in the present study,

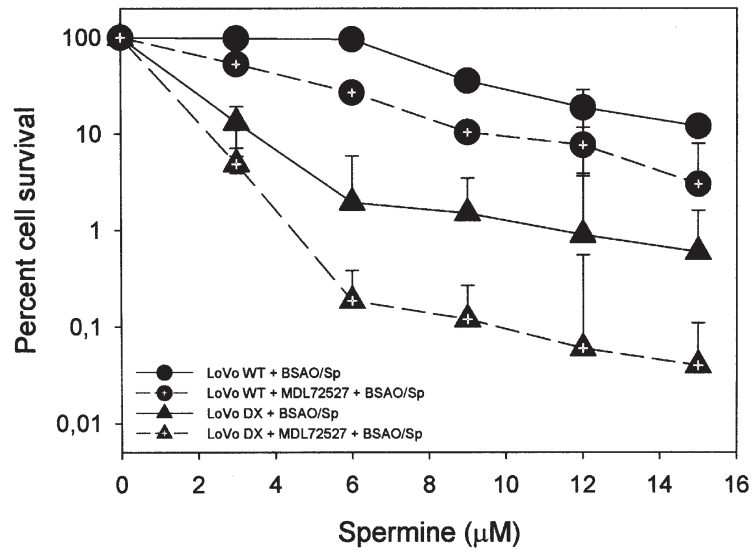

Figure 3. Effect of spermine concentration in the presence of BSAO on the survival of LoVo WT and LoVo DX cells. Dose-effect relationship of plating efficiency for 24-h pre-incubation with $300 \mu \mathrm{M}$ MDL 72527 at $37^{\circ} \mathrm{C}$. Each point is the mean value of the results of three or four experiments with 2-5 samples per treatment. The error bars indicate \pm SD. When not shown, SD is smaller than the symbols.

that exposure of cells to either BSAO or spermine alone (at the concentration range applied in this work) has no significant effect on cell viability.

In Fig. 2, the dose-response curve is shown for 24-h exposure to MDL 72527. In agreement with other colon adenocarcinoma cells (16), the sensitivity of LoVo cells to this drug is low. MDL 72527 at $300 \mu \mathrm{M}$ was the highest concentration, which showed no significant cytotoxic effect to LoVo WT cells, while MDR cells (LoVo DX) were only slightly more sensitive. This concentration was chosen for all following experiments. In the presence of a constant activity of BSAO $\left(6.54 \times 10^{-3} \mathrm{IU} / \mathrm{ml}\right)$, increasing concentrations of spermine improved cytotoxicity (Fig. 3). The dose-response curves are biphasic in the semilogarithmic plots. At spermine concentrations of below $6 \mu \mathrm{M}$, the curve slopes are considerably steeper than at higher concentrations, where the slope appears to be the same for all cells and treatments. LoVo DX cells were not only more sensitive to the oxidation products of spermine but MDL 72527 also had a considerably greater effect on their survival than on the survival of LoVo WT cells. Cell survival was lower by approximately one log unit, compared with cells that had not been pre-treated with the polyamine oxidase inactivator. In Fig. 4, time-effect relationships are shown. In this and related experiments, the cells were pre-incubated with $300 \mu \mathrm{M}$ MDL 72527 for 6,24 or $48 \mathrm{~h}$, and were then exposed to $6 \mu \mathrm{M}$ spermine and BSAO for $1 \mathrm{~h}$, except for the curves with empty symbols, which represent the effect of MDL 72527 in the absence of BSAO and spermine. Again, the MDR cells were considerably more sensitive to the treatment than the wild-type cells. However, when the pre-incubation time with MDL 72527 was prolonged to $48 \mathrm{~h}$, the percentage of surviving cells was higher by one order of magnitude (Fig. 4C) as compared with cells exposed to this drug for only $24 \mathrm{~h}$ (Fig. 4B). The increase in spermine concentration from 6 to $12 \mu \mathrm{M}$ had a marked effect on cell survival (not shown). It should be noted that all spermine concentrations are $<25.5 \mu \mathrm{M}$, the $\mathrm{Km}$ of spermine for BSAO (9). Therefore, the rate of hydrogen peroxide formation during the incubation period of $60 \mathrm{~min}$ is not constant. From the 

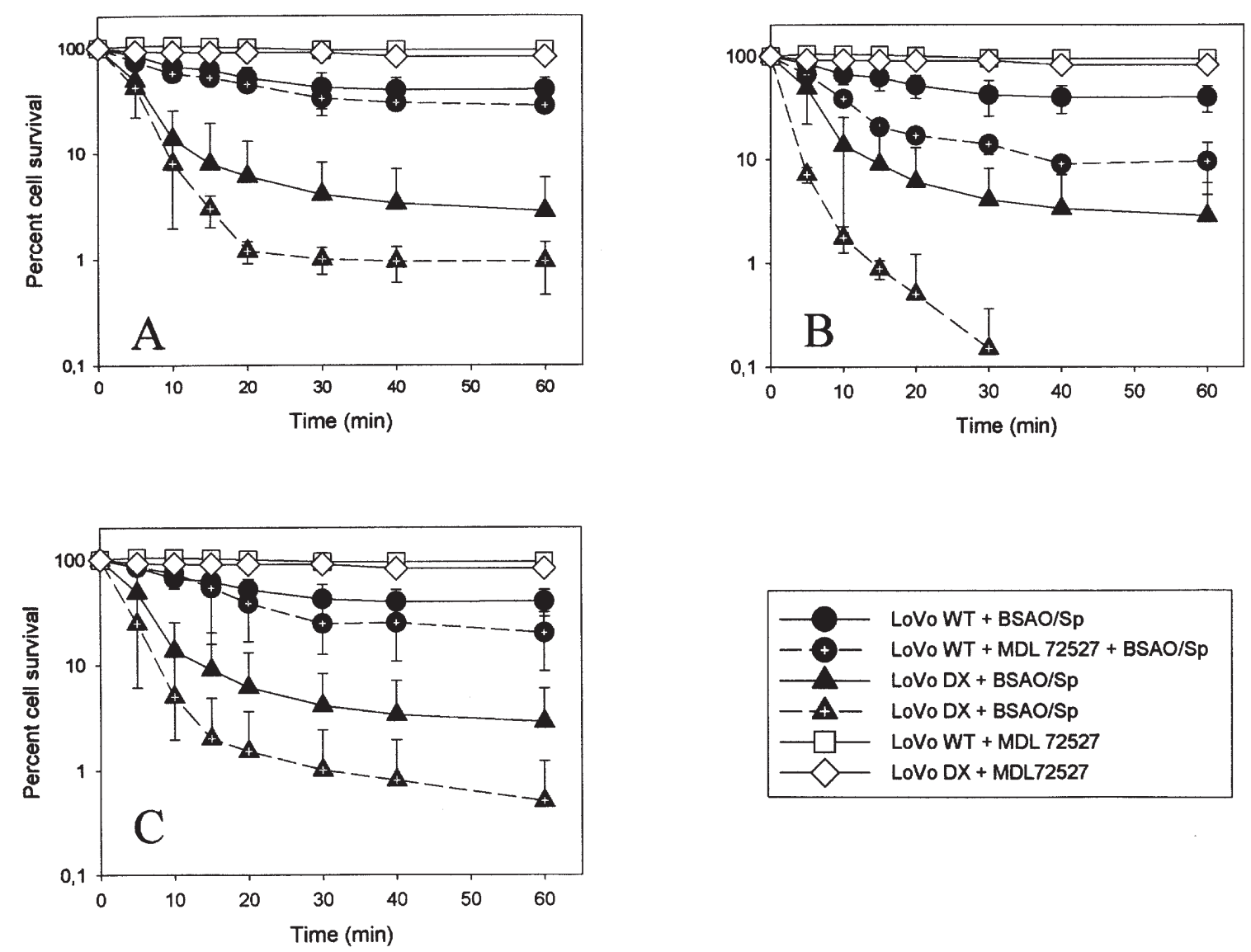

Figure 4. Effect of pre-treatment with MDL 72527 (followed by exposure to BSAO and spermine) on the survival of LoVo WT and LoVo DX cells in function of pre-incubation time. A, pre-incubation with $300 \mu \mathrm{M}$ MDL 72527 for $6 \mathrm{~h}$ at $37^{\circ} \mathrm{C}$. B, pre-incubation with $300 \mu \mathrm{M}$ MDL 72527 for $24 \mathrm{~h}$. C, preincubation with $300 \mu \mathrm{M}$ MDL 72527 for $48 \mathrm{~h}$. Pre-incubations were followed by 60 -min exposure to BSAO $\left(6.54 \times 10^{-3} \mathrm{IU}\right)$ and $6 \mu \mathrm{M}$ spermine. The curves with the empty symbols represent incubations with MDL 72527 in the absence of BSAO and spermine. Each point is the mean value of the results of three or four independent experiments with 2-5 samples per treatment. The error bars indicate $\pm \mathrm{SD}$. When not shown, SD is smaller than the symbols.

results illustrated in Figs. 3 and 4, the following facts become obvious: i) The plating efficiency decreases with incubation time with BSAO and with concentration of spermine. ii) In agreement with previous results, MDR cells were more sensitive to treatment with BSAO and spermine than their wild-type counterparts. iii) Pre-incubation of both, LoVo WT and LoVo DX cells, with MDL 72527 increases the sensitivity of these cells considerably to toxic polyamine metabolites.

Since the prolongation of the pre-incubation time with MDL 72527 to over $24 \mathrm{~h}$ decreased the sensitivity to treatment with BSAO and spermine, experiments were carried out with only 12- and 6-h pre-incubations with MDL 72527. With these incubation times, the sensitization of the cells to subsequent exposure to BSAO and spermine was considerably weaker than at $24 \mathrm{~h}$. In Fig. 4A, the results obtained for 6-h pre-incubation with MDL 72527 are illustrated.

Ultrastructural effects of exposure to MDL 72527 on LoVo cells. Under the treatment conditions described in the previous section, both LoVo WT and LoVo DX cells exhibited only minor phenotypic changes, as judged from scanning electron micrographs (not shown). However, transmission electron micrographs revealed a number of morphological changes, which are indicative of the cytotoxic effect of the treatment. The ultrastructure of untreated controls of LoVo WT (Fig. 5a and b) and LoVo DX cells (Fig. 5c and d) was similar, with roundish nuclei, a compact cytoplasmic matrix and mitochondria with condensed matrix and parallel cristae. However, even at $6 \mathrm{~h}$ after exposure to $300 \mu \mathrm{M}$ MDL 72527, LoVo WT (Fig. 6a and b) and LoVo DX (Fig. 6c and d) cells had formed numerous, large cytoplasmic vacuoles; the drug-resistant cells showed less vacuoles than the wild-type cells. This difference may be caused by the high activity of the P-glycoprotein pump in the MDR cells. However, whether MDL 72527 is a substrate of this pump has not yet been established. In some cells, the shape of the nucleus was affected by the extensive vacuolization. Some of these vacuoles appeared empty, while most of them contained differently structured material, such as small electron-dense tufts, granular bodies with a punctate pattern, and lysosome-like formations. The vacuoles do not seem to be delimited by a membrane, but rather appear as a rarefaction of the cytoplasmic matrix. No significant structural change of the mitochondria were seen in the wild-type cells (Fig. 6b), while the mitochondria of the MDR-resistant cells showed an irregular morphology with swelling of the cristae (Fig. 6d), and the cytoplasmic matrix exhibited many rarefactions.

Surprisingly, the vacuoles in both cell lines decreased in number and size after $24 \mathrm{~h}$ of incubation with MDL 72527 , and disappeared nearly completely upon prolongation of the treatment with MDL 72527 to $48 \mathrm{~h}$, and the cells resumed a 

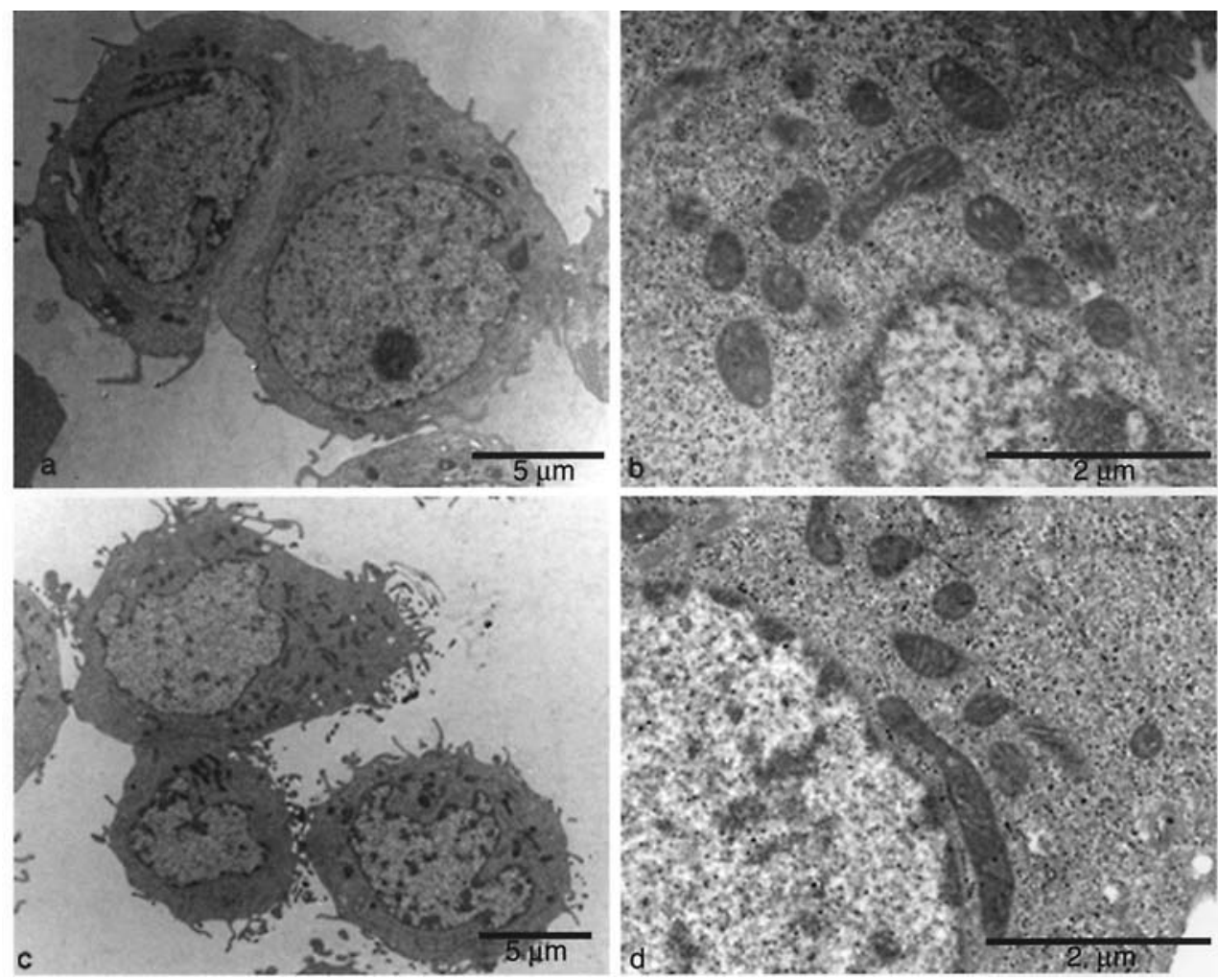

Figure 5. Ultrastructural morphology of LoVo cells. a and b, untreated wild-type (LoVo WT) cells; c and d, untreated multidrug-resistant (LoVo DX) cells.
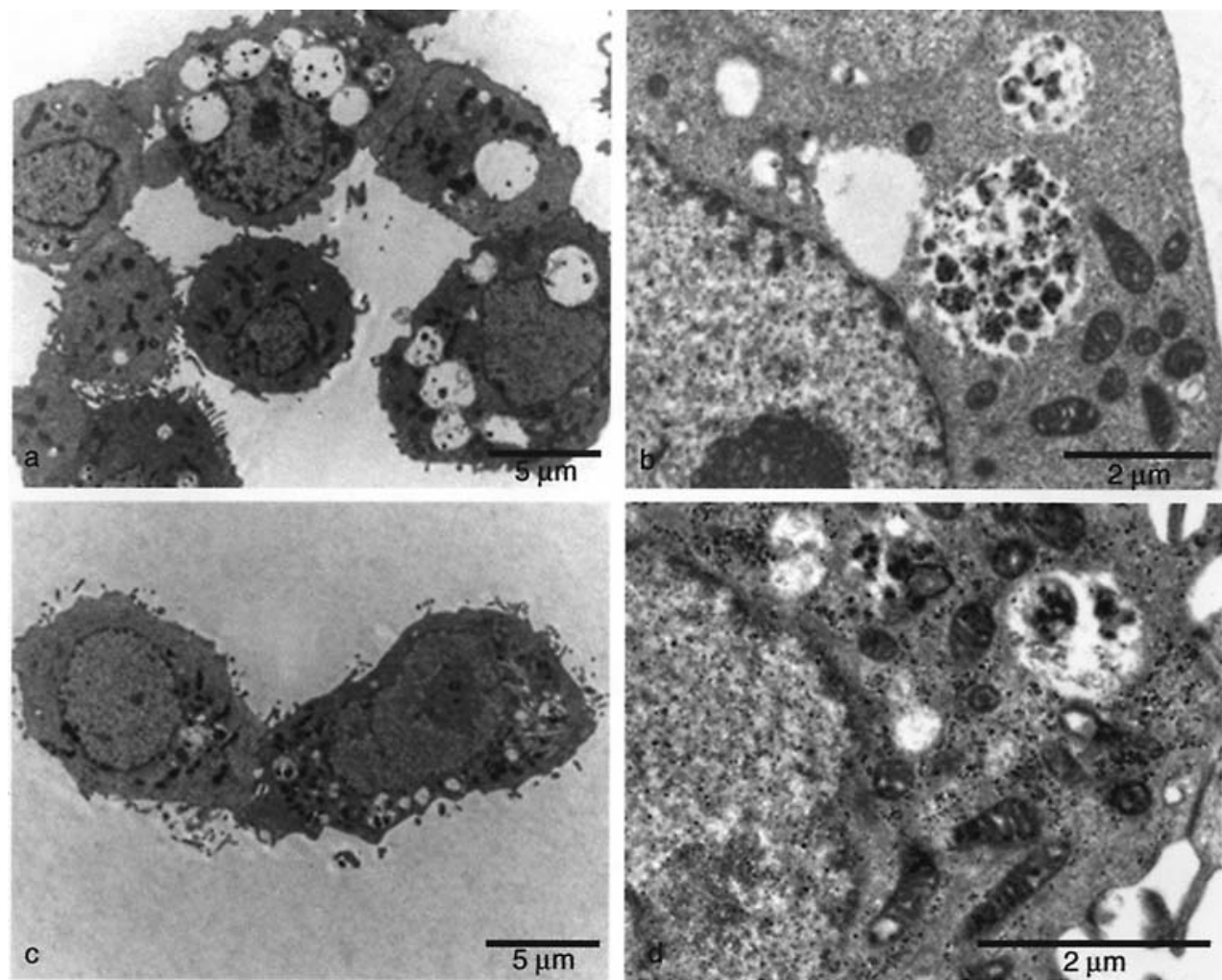

Figure 6. Pre-incubation of LoVo cells with $300 \mu \mathrm{M}$ MDL 72527 for $6 \mathrm{~h}$ at $37^{\circ} \mathrm{C}$ : effect on ultrastructural morphology. a and b, wild-type (LoVo WT) cells; $\mathrm{c}$ and d, multidrug-resistant (LoVo DX) cells.

normal appearance (Fig. 7a and c). However, at a high magnification, alterations in some mitochondria of the LoVo DX cells (Fig. 7d) and, in both cell lines, aggregates of structures embedded in the cytoplasmic matrix, similar to those observed inside the vacuoles after 6-h treatment (Fig. 7a-c), were observed. 

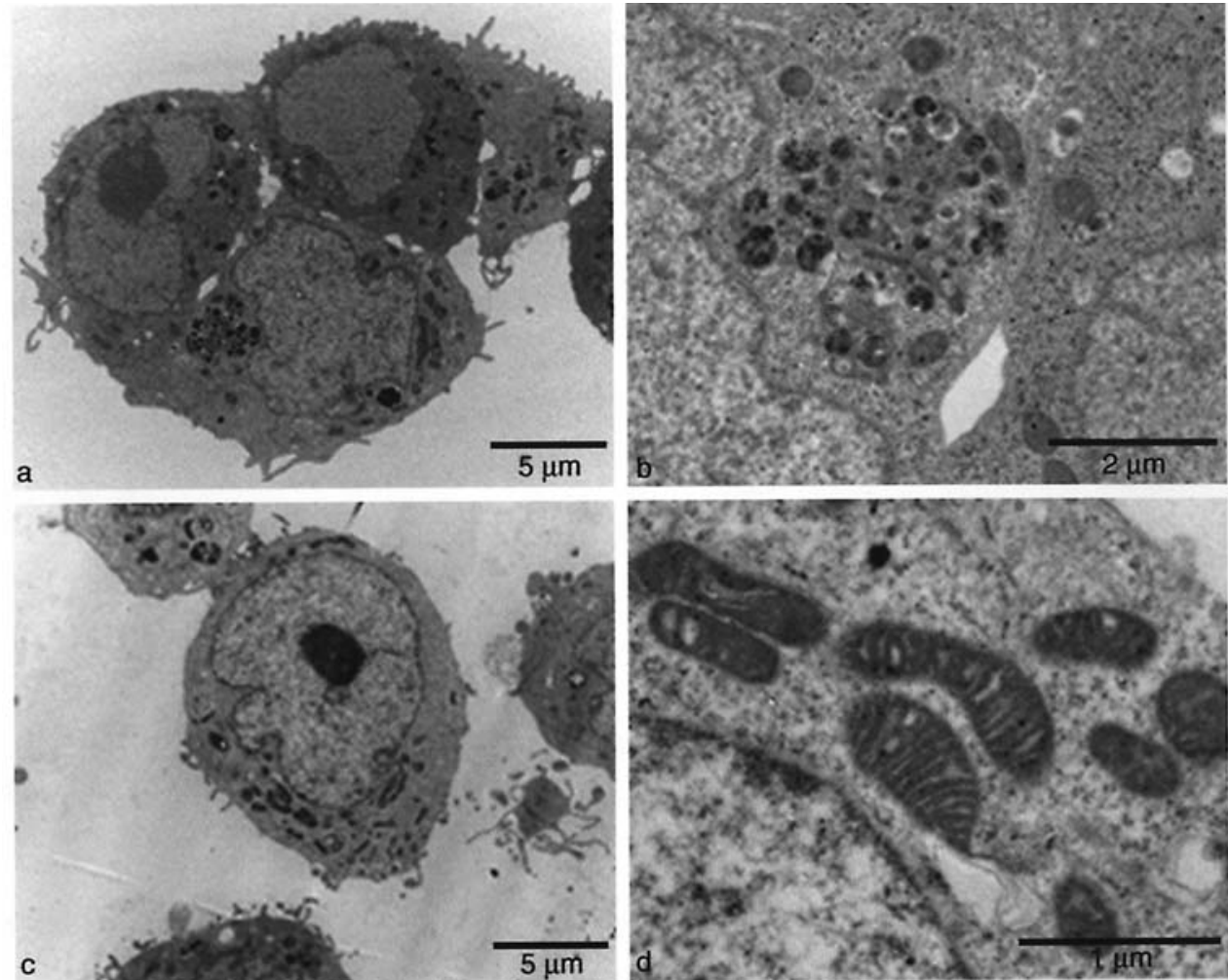

Figure 7. Pre-incubation of LoVo cells with $300 \mu \mathrm{M}$ MDL 72527 for $24 \mathrm{~h}$ at $37^{\circ} \mathrm{C}$; effect on ultrastructural morphology. a and b, wild-type (LoVo WT) cells; $\mathrm{c}$ and d, multidrug-resistant (LoVo DX) cells.
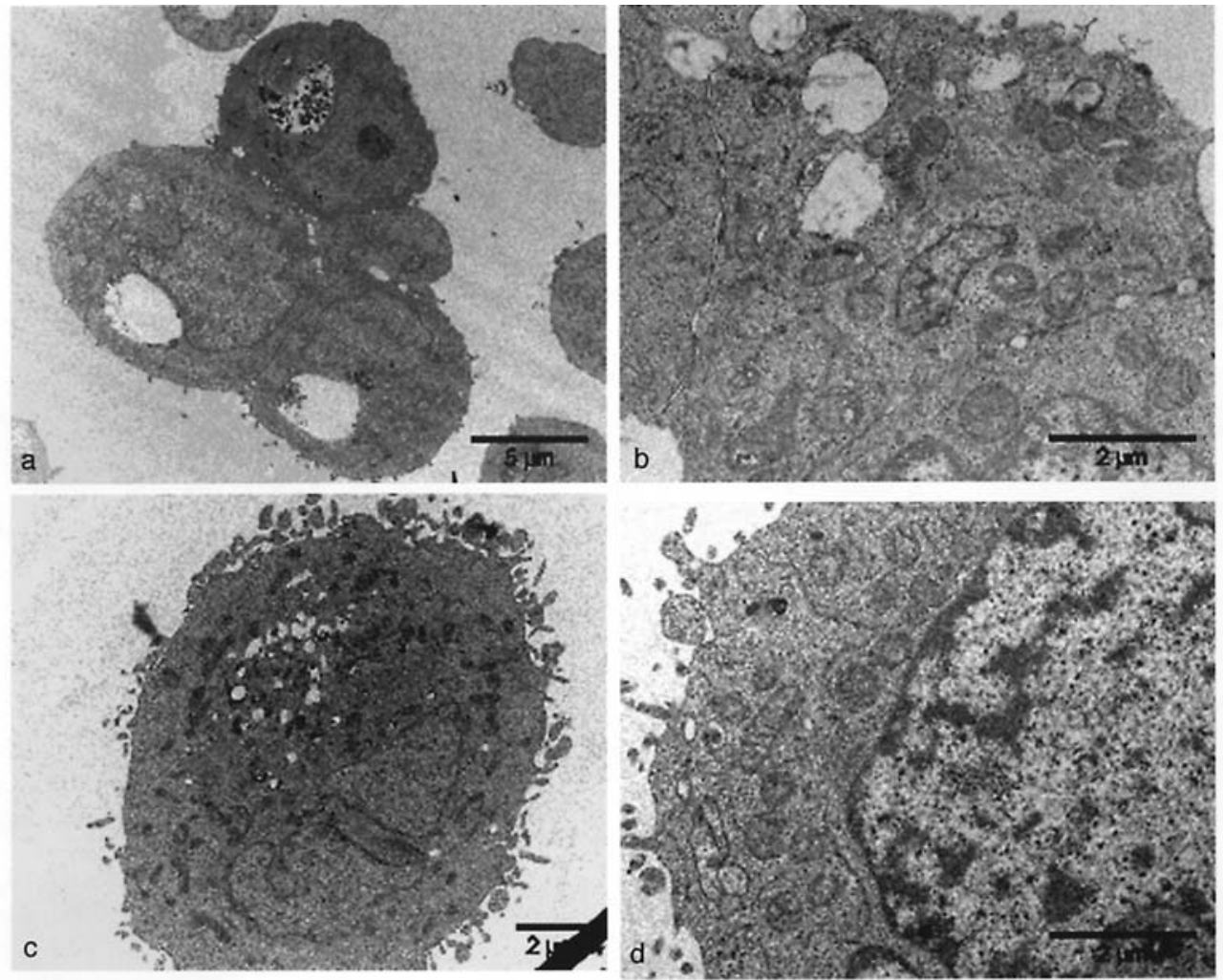

Figure 8. Effect of exposure of LoVo cells to $300 \mu \mathrm{M}$ MDL 72527 for $6 \mathrm{~h}$, followed by treatment for $60 \mathrm{~min}$ (at $37^{\circ} \mathrm{C}$ ) with BSAO and $6 \mu \mathrm{M}$ spermine, on the ultrastructural morphology. a and b, wild-type (LoVo WT) cells; c and d, multidrug-resistant (LoVo DX) cells.

In agreement with previous observations (3), treatment for $1 \mathrm{~h}$ with BSAO and spermine alone produced no significant morphological modifications in LoVo WT cells, while consistent alterations of the mitochondrial shape, i.e. swelling 

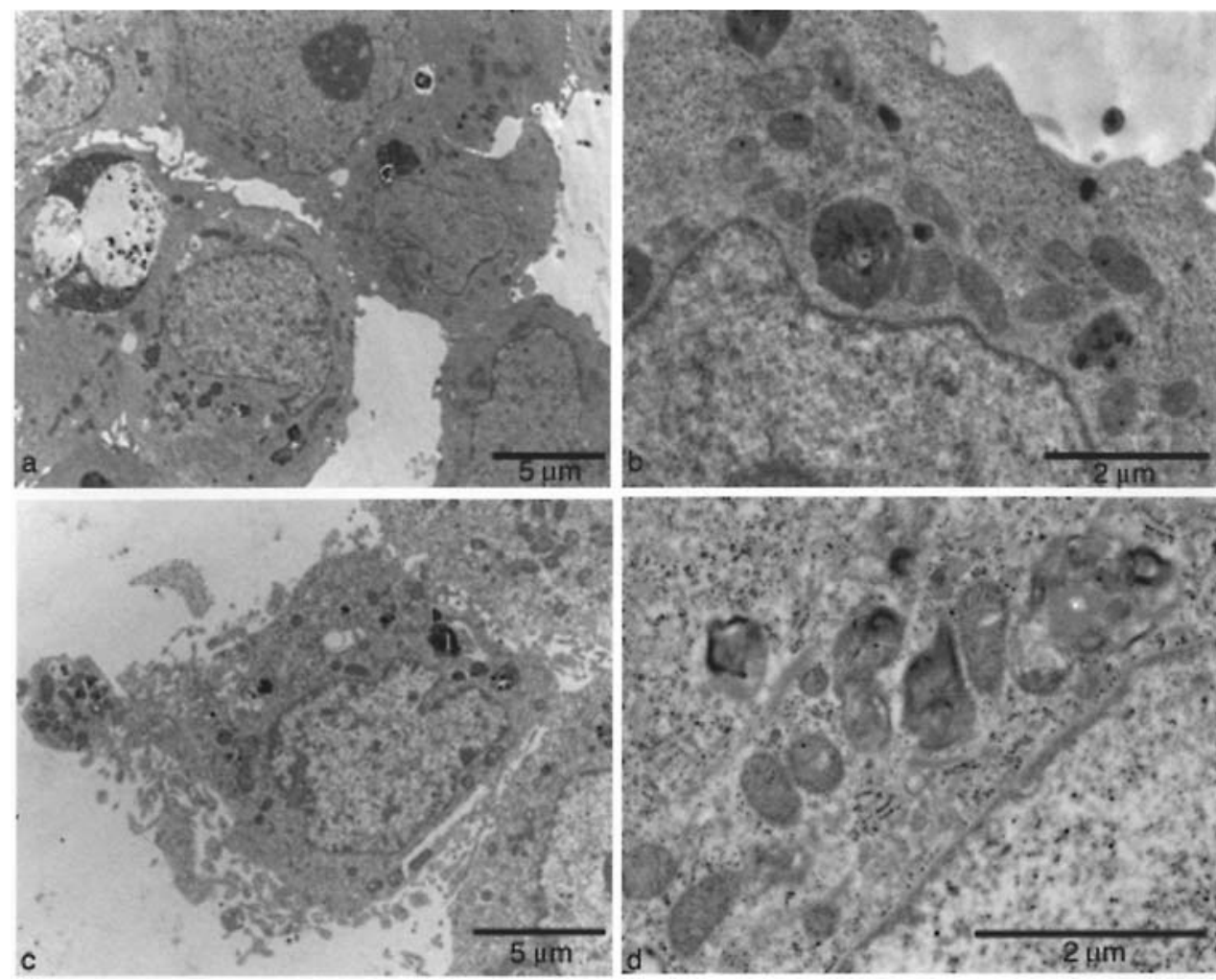

Figure 9. Effect of exposure of LoVo cells to $300 \mu \mathrm{M}$ MDL 72527 for $24 \mathrm{~h}$, followed by treatment for $60 \mathrm{~min}$ (at $37^{\circ} \mathrm{C}$ ) with BSAO and $6 \mu \mathrm{M}$ spermine, on the ultrastructural morphology. a and b, wild-type (LoVo WT) cells; c and d, multidrug-resistant (LoVo DX) cells.

and disappearance of cristae, were observed in LoVo DX cells. Pre-treatment with $300 \mu \mathrm{M}$ MDL 72527 for $6 \mathrm{~h}$ accentuated the effect of BSAO and spermine on both cell lines (Fig. 8), but MDR cells were more affected (Fig. 8c and $\mathrm{d}$ ). With prolongation of the pre-incubation time with $300 \mu \mathrm{M}$ MDL 72527 to $24 \mathrm{~h}$, followed by treatment with BSAO and spermine, the mitochondrial damage and the general impairment of cell morphology paralleled the decline in survival time. The mitochondrial damage is particularly obvious from the electron micrographs (Fig. 9b and d).

In summary, comparison of the electron micrographs taken from LoVo WT and LoVo DX cells demonstrates that the higher sensitivity of the latter cells to toxic polyamine metabolites is paralleled by more pronounced morphological alterations, both of cytoplasmic and mitochondrial structures. However, this was not the case with the formation of cytoplasmic vacuoles, which did not parallel the toxic effect of BSAO and spermine that followed pre-incubation with MDL 72527: with 24 h of pre-treatment, the survival of LoVo cells was higher than with 6- or 48-h pre-incubation, while the size and number of cytoplasmic vacuoles was greater with $6 \mathrm{~h}$ of pre-incubation than with longer pre-incubation times.

\section{Discussion}

The lack of tumor specificity with cytotoxic drugs and the development of MDR are major problems of conventional cancer chemotherapy. The in situ formation of toxic compounds by an enzymatic reaction is a promising start, particularly because MDR cells appear significantly more sensitive to toxic polyamine metabolites than corresponding wild-type cells, as shown in the present study. The higher sensitivity of LoVo DX cells to cytotoxic spermine metabolites has been attributed to mitochondrial membrane depolarization and an elevated basal production of reactive oxygen species (3).

The development of MDR is associated with a number of phenotypic alterations. MDR cancer cells usually display a decreased intracellular drug accumulation and/or drug distribution $(17,18)$ due to the overexpression of genes which encode membrane-bound transporter proteins, such as a $170-\mathrm{kDa} \mathrm{P}$-glycoprotein. This glycoprotein functions as an energy-dependent pump that expels drugs out of cells (19).

Prevention of accumulation of the toxic products of spermine oxidation by MDR cells appears to play a minor role because LoVo DX cells were significantly more sensitive than LoVo WT cells to exposure to BSAO and spermine. For the slow release of toxic spermine metabolites in tumors, the use of BSAO conjugated to biocompatible polymers has been considered $(4,20)$. The high polyamine content of tumor cells and the release of polyamines from damaged cells may make the administration of spermine superfluous in therapeutic application.

MDL 72527 is highly toxic to transformed hematopoietic cells (11) but is considerably less toxic to colon adenocarcinoma cells $(16,21)$, as confirmed by the present results. Since leukemia cells have a lower glutathione content (22) than SW 620 colon adenocarcinoma cells (23), i.e. their intrinsic defense system to oxidative stress is considerably weaker, differences in glutathione content may partly explain the difference in sensitivity to MDL 72527 between leukemia and adenocarcinoma cell lines. However, this explanation is not relevant to the difference in sensitivity between LoVo 
WT and LoVo DX cells because their glutathione content is the same (3). We believe that the difference in mitochondrial electron transport chain activity is the main reason for their difference in sensitivity to oxidative stress.

In the present study, an emphasis was placed on the ability of MDL 72527 to sensitize LoVo cells to BSAO/ spermine-induced toxicity. There was an obvious timedependence for optimum sensitization. The lowest cell survival was observed with $24 \mathrm{~h}$ of pre-incubation with MDL 72527. Under these conditions, a loss of both LoVo WT and LoVo DX cells was greater by approximately one log unit in comparison with non-sensitized cells. At both an elongated and shorter exposure to MDL 72527, cell loss was lower.

Among the morphological changes produced by MDL 72527 , the rapid formation of cytoplasmic vacuoles was the most striking. These vacuoles are most probably of lysosomal origin. A diamine, structurally related to MDL 72527 (2,5diamino-3-hexyne) (24), and the known lysosomotropic compound, chloroquine (11), cause the formation of very similar vacuoles in leukemia cells. In contrast to the vacuoles in leukemia cell lines, the vacuoles in LoVo cells did not coalesce to form larger vacuoles with time but, on the contrary, they disappeared nearly completely in the presence of MDL 72527 with 48 h of incubation. However, in the present study, incubation with MDL 72527 was limited to $48 \mathrm{~h}$. It is not excluded that, at prolonged exposure to this drug, vacuoles may reappear in LoVo cells since, in other colon adenocarcinoma-derived cell lines (SW620 and SW480), long-term exposure to MDL 72527 produced huge cytoplasmic vacuoles (16).

Vacuole formation did not correlate directly with loss of cell viability. In fact, vacuole formation was more extensive in LoVo WT cells than in MDR cells, and sensitization to treatment with BSAO and spermine was optimal with $24 \mathrm{~h}$ of pre-incubation. A likely explanation for the discrepancy between the rapid formation of cytoplasmic vacuoles and the effect of the treatment on cell viability is that the lysosomotropic effect of MDL 72527 is maximal within less than $24 \mathrm{~h}$, whereas compensatory reactions start presumably with the impairment of cell metabolism and recover physiological function to some extent in the presence of the drug for only a limited period. It is known that oxidative stress stimulates defense mechanisms by inducing enzymes which assist in antagonizing oxidative damage (25).

Regarding the impairment of cell viability, the morphological alterations of the mitochondrial structure seem more relevant than the formation of cytoplasmic vacuoles, since they were more accentuated in the drug-resistant cells than in the wild-type cells. Moreover, they seem to be paralleled by the loss of cell viability. It is known from previous study (7) that one of the earliest signs of cell damage by BSAO and spermine is the depolarization of the mitochondrial membrane.

In conclusion, sensitization of LoVo cells to hydrogen peroxide, the main cytotoxic agent in the present experimental setting, (3) is most probably caused by the lysosomotropic effect of MDL 72527. 3-Aminopropanal, according to Houen et al (26), a major product of spermine oxidation by BSAO (Fig. 1), is a known lysosomotropic compound that causes oxidative stress due to the release of lysosomal enzymes $(27,28)$. There is an obvious analogy between the cytotoxic effect of 3-aminopropanal and treatment with MDL 72527, followed by BSAO-catalyzed spermine oxidation. While 3aminopropanal combines lysosomotropic effect and aldehyde function in one molecule, the lysosomotropic effect in the combination treatment is provided by MDL 72527 (11). During the subsequent treatment with BSAO and spermine, hydrogen peroxide is expected to amplify the oxidative stress that was already initiated by MDL 72527, while 3-aminopropanal and the other spermine-derived aldehydes exert toxicity based on their chemical reactivity and their lysosomotropy. An important role of lysosomes in both necrotic and apoptotic cell death is well founded (29). Considering this, it is of interest to note that hydrogen peroxide cytotoxicity has been reported to be preceded by damage of lysosomal membranes (30). Owing to its lysosomotropic effect, pre-treatment with MDL 72527 amplifies the ability of the metabolites formed from spermine by oxidative deamination to induce cell death. In a recent review (31), lysosomes were denoted as the Achilles heel of cancer cells. Our results support this. Since it is conceivable that combined treatment with a lysosomotropic compound and $\mathrm{BSAO} /$ spermine would be effective in tumors, it is of interest to search for lysosomotropic compounds which are suitable for application in a therapeutic setting.

The method of enzyme catalyzed-formation of hydrogen peroxide and of other toxic products may be considered as a treatment analogous to the use of irradiation techniques. Both methods are designed to produce radicals in a narrow, defined area over an extended period of time. It is our hope that this approach will open a new avenue in combating cancer and in treating MDR cancer patients.

\section{Acknowledgements}

We are grateful to the University of Rome 'La Sapienza' for their support in the form of a grant to N. Seiler as a visiting Professor in the Department of Biochemical Sciences 'A. Rossi-Fanelli' in 2005. This work was partially supported by the Ministero della Salute (1\% Fondo Sanitario Nazionale), the Italian MIUR (Ministero dell'Istruzione, dell'Università e della Ricerca) and by funds MIUR-PRIN 2003 and 2005 (Cofin) (EA).

\section{References}

1. Cohen SS: A guide to the Polyamines. Oxford University Press, New York, Oxford, 1998.

2. Seiler N and Raul F: Polyamines and apoptosis. J Cell Mol Med 9: 623-642, 2005.

3. Calcabrini A, Arancia G, Marra M, Crateri P, Befani O, Martone A and Agostinelli E: Enzymatic oxidation products of spermine induce greater cytotoxic effects on human multidrugresistant colon carcinoma cells $(\mathrm{LoVO})$ than on their wild-type counterparts. Int J Cancer 93: 43-52, 2002.

4. Averill-Bates DA, Cherif A, Agostinelli E, Tanel A and Fortier G: Anti-tumoral effect of native and immobilized bovine serum amine oxidase in a mouse melanoma model. Biochem Pharmacol 69: 1693-1704, 2005.

5. Yoshikawa T, Kokura S, Tainaka K, Naito Y and Kondo M: A novel cancer therapy based on oxygen radicals. Cancer Res 55: 1617-1620, 1995.

6. Grandi M, Geroni C and Giuciani FC: Isolation and characterization of the human colon adenocarcinoma cell line resistant to doxorubicin. Br J Cancer 54: 515-518, 1986.

7. Arancia G, Calcabrini A, Marra M, Crateri P, Artico M, Martone A, Martelli F and Agostinelli E: Mitochondrial alterations induced by serum amine oxidase and spermine on human multidrug resistant tumor cells. Amino Acids 26: 273-282, 2004. 
8. Droege W: Free radicals in the physiological control of cell function. Physiol Rev 82: 47-96, 2002.

9. Averill-Bates DA, Agostinelli E, Przybytkowski E, Mateescu MA and Mondovi B: Cytotoxicity and kinetic analysis of purified bovine serum amine oxidase in the presence of spermine in Chinese hamster ovary cells. Arch Biochem Biophys 300: 75-79, 1993.

10. Seiler N, Duranton B and Raul F: The polyamine oxidase inactivator MDL 72527. Prog Drug Res 59: 1-40, 2002.

11. Dai H, Kramer DL, Yang C, Murti KG, Porter CW and Cleveland JL: The polyamine oxidase inactivator MDL-72,527 selectively induces apoptosis in transformed hematopoietic cells through lysosomotropic effects. Cancer Res 59: 4944-4955, 1999.

12. Turini P, Sabatini S, Befani O, Chimenti F, Casanova C, Riccio P and Mondovi B: Purification of serum amine oxidase. Anal Biochem 125: 294-298, 1982.

13. Bey P, Bolkenius FN, Seiler N and Casara P: N-2,3-Butadienyl1,4-butanediamine derivatives: Potent irreversible inhibitors of mammalian polyamine oxidase. J Med Chem 28: 1-2, 1985.

14. Dolfini E, Dasdia T, Arancia G, Molinari A, Calcabrini A, Scheper RJ, Flena MJ, Gariboldi MB and Monti E: Characterization of a clonal human colon adenocarcinoma line intrinsically resistant to doxorubicin. Br J Cancer 76: 67-76, 1997.

15. Molinari A, Calcabrini A, Crateri P and Arancia G: Interaction of anthracycline antibiotics with cytoskeletal components of cultured carcinoma cells (CG5). Exp Mol Pathol 53: 11-33, 1990.

16. Duranton B, Holl V, Schneider Y, Carnesecchi S, Gosse F, Raul $\mathrm{F}$ and Seiler N: Cytotoxic effects of the polyamine oxidase inactivator MDL 72527 to two human colon carcinoma cell lines SW 480 and SW 629. Cell Biol Toxicol 18: 381-396, 2002.

17. Arancia G, Calcabrini A, Meschini S and Molinari A: Intracellular distribution of anthracycline in drug resistant cells. Cytotechnology 27: 95-111, 1998.

18. Gervasoni JE, Fields SZ, Krishna S, Baker MA, Rosado M, Thuraisami K, Hinderburg AA and Taub RN: Subcellular distribution of doxorubicin in P-glycoprotein positive and negative drug resistant cell lines using laser-assisted confocal microscopy. Cancer Res 51: 4955-4963, 1991.

19. Gottesman MM and Pastan I: Biochemistry of multidrug resistance mediated by the multidrug transporter. Annu Rev Biochem 62: 385-427, 1993.
20. Demers N, Agostinelli E, Averill-Bates DA and Fortier G: Immobilization of native and poly(ethylene glycol)-treated ('PEGylated') bovine serum amine oxidase into a biocompatible hydrogel. Biotechnol Appl Biochem 33: 201-207, 2001.

21. Seiler N, Renault J, Gosse F, Roussi S and Raul F: Cytotoxicity of the polyamine oxidase inactivator MDL 72527 to cancer cells: comparison with a saturated structural analogue. Int J Oncol 27: 1669-1676, 2005.

22. Tagliabue G, Pifferi A, Balconi G, Mascellari E, Geroni C, D'Incalci $\mathrm{M}$ and Ubezio P: Intracellular glutathione heterogeneity in L1210 murine leukemia sublines made resistant to DNAinteracting antineoplastic agents. Int J Cancer 54: 435-442, 1993.

23. Chau YP, Shiah SG, Don MJ and Kuo ML: Involvement of hydrogen peroxide in topoisomerase inhibitor B-lapachoneinduced apoptosis and differentiation of human leukaemia cells. Free Radic Biol Med 24: 660-670, 1998

24. Porter CW, Stanek J, Black J, Vaughan M, Ganis B and Pleshkewych A: Morphological evidence for an apparent lysosomotropic activity by unsaturated putrescine analogues. Cancer Res 50: 1929-1935, 1990.

25. Greenberger JS, Kagan VE, Pearce L, Boriseniao G, Tyurina Y and Epperli MW: Modulation of redox signal transduction pathways in the treatment of cancer. Antioxid Redox Signal 3: 347-359, 2001.

26. Houen G, Bock K and Jensen AL: HPLC and NMR investigation of the serum amine oxidase-catalyzed reaction of polyamines. Acta Chem Scand 48: 52-60, 1994.

27. Zhao M, Antunes F, Eaton JW and Brunk UT: Lysosomal enzymes promote mitochondrial oxidant production, cytochrome c release and apoptosis. Eur J Biochem 270: 13778-13786, 2003.

28. Yu Z, Li W and Brunk UT: 3-Aminopropanal is a lysosomotropic aldehyde that causes oxidative stress and apoptosis by rupturing lysosomes. APMIS 111: 643-652, 2003

29. Guicciardi ME, Leist M and Gores GJ: Lysosomes in cell death. Oncogene 23: 2881-2890, 2004.

30. Zdolsek J, Zhang H, Roberg K and Brunk UT: $\mathrm{H}_{2} \mathrm{O}_{2}$-mediated damage to lysosomal membranes of J-774 cells. Free Rad Res Commun 18: 171-185, 1993.

31. Fehrenbacher $\mathrm{N}$ and Jaattela M: Lysosomes as targets for cancer therapy. Cancer Res 103: 1551-1560, 2005. 